selected, leading to possible confounding by indication, which in turn makes ETN look protective. Researchers should take these selection biases into account when analysing their results.

Disclosure of Interest: None declared

DOI: 10.1136/annrheumdis-2018-eular.5211

SATURDAY, 16 JUNE 2018

Gut bacteria: the boss of the immune system

\section{OP0352 AN INCREASED PREVALENCE OF PERIODONTAL DISEASE, PORPHYROMONAS GINGIVALIS AND AGGREGATIBACTER ACTINOMYCETEMCOMITANS IN ANTI-CCP POSITIVE INDIVIDUALS AT-RISK OF INFLAMMATORY ARTHRITIS}

K. Mankia ${ }^{1}$, Z. Cheng ${ }^{2}$, T. Do ${ }^{2}$, J. Kang ${ }^{2}$, L. Hunt ${ }^{1}$, J. Meade ${ }^{2}$, V. Clerehugh ${ }^{3}$ A. Speirs ${ }^{3}$, A. Tugnait ${ }^{3}$, E. Hensor ${ }^{4}$, D. Devine ${ }^{2}$, P. Emery ${ }^{1}{ }^{1}$ Rheumatology, Leeds Institute of Rheumatic and Musculoskeletal Medicine and NIHR Leeds Biomedical Research Centre; ${ }^{2}$ Oral Microbiology, University of Leeds; ${ }^{3}$ Leeds Dental Institute, Leeds Teaching Hospitals Trust, ${ }^{4}$ Rheumatology, Leeds Institute of Rheumatic and Musculoskeletal Medicine, Leeds, UK

Background: The prevalence of periodontal disease is increased in RA, and periodontitis is associated with the bacterium Porphyromonas gingivalis $(\mathrm{Pg})$, which can citrullinate arginine residues. ${ }^{1}$ These observations suggest periodontitis may be a key initiator of RA-related autoimmunity. Importantly, clinical periodontal disease, and the relative abundance of periodontal bacteria have not been described in seropositive individuals at risk of developing RA who do not have synovitis.

Objectives: To investigate the prevalence of periodontal disease and the relative abundance of key periodontal bacteria in anti-CCP positive at-risk individuals without synovitis.

Methods: Anti-CCP positive individuals with musculoskeletal symptoms but no clinical synovitis $(\mathrm{CCP}+)$, early RA (RA) patients and healthy controls $(\mathrm{HC})$ were recruited. CCP +underwent a 38 joint ultrasound (US) assessment. Periodontal examination was performed by a dentist; six sites per tooth were assessed for clinical attachment level (CAL), pocket depth (PD) and bleeding on probing (BOP). Periodontal disease sites (PDD) were defined as $C A L>2 \mathrm{~mm}$ and $P D>4 \mathrm{~mm}$. A clinical consensus was agreed for each case by three dentists. DNA, isolated from subgingival plaque from diseased and healthy periodontal sites, was pairend sequenced (Illumina HiSeq3000). Taxonomic and functional profiles were obtained from MG-Rast and differences between groups studied using DESeq2. Mann-Whitney $\mathrm{U}$ tests were used to compare groups and Spearman Rho used for correlations. For metagenomic data, Wald test was used to compare relative abundance.

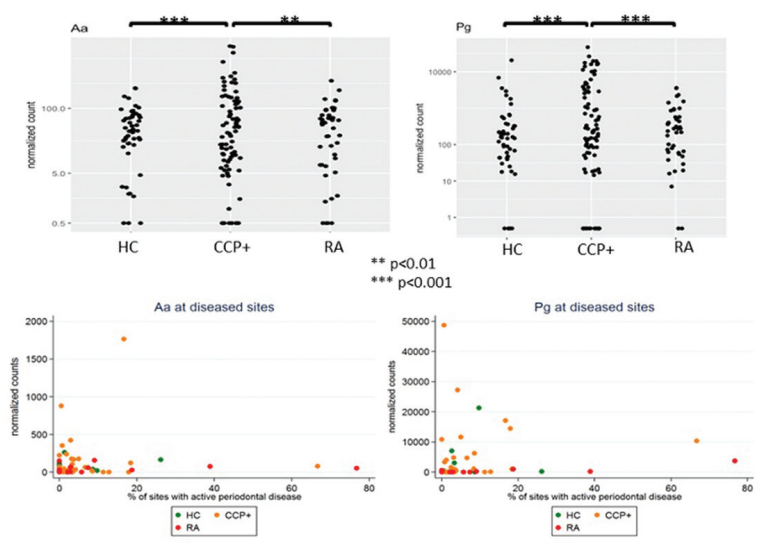

Abstract OP0352 - Figure 1 Relative abundance of Aggregatibacter actinomycetemcomitans $(\mathrm{Aa})$ and Porphyromonas gingivalis $(\mathrm{Pg})$ according to RA status and extent of periodontal disease

Results: $48 \mathrm{CCP}+, 26 \mathrm{RA}$ and $32 \mathrm{HC}$ were recruited. Groups were balanced for age, sex and smoking. All but 2 (96\%) CCP thad no US synovitis (greyscale $\geq 1$ and power Doppler $\geq 1$ ). Dentists classified $73 \% \mathrm{CCP}+, 38 \% \mathrm{HC}$ $(p=0.02)$ and $54 \%$ RA as having clinical periodontitis. The percentage of periodontal sites with $\mathrm{CAL}>2 \mathrm{~mm}, \mathrm{PD}>4 \mathrm{~mm}, \mathrm{BOP}, \mathrm{PDD}$ and active PDD (PDD +BOP) were all greater in $\mathrm{CCP}+$ compared to $\mathrm{HC}(\mathrm{p}<0.05)$ and similar to $\mathrm{RA}$. In nonsmokers, PDD and active PDD were more prevalent in $\mathrm{CCP}+$ compared to $\mathrm{HC}$. Metagenomic data indicated $\mathrm{CCP}$ +had increased relative abundance of both $\mathrm{Pg}$ and Aggregatibacter actinomycetemcomitans $(A a)$ compared to $\mathrm{HC}(\mathrm{p}<0.001)$ and RA $(\mathrm{p}<0.01)$. However, clinical periodontitis was only associated with increased relative abundance of $P g(\mathrm{p}<0.001)$ but not $A a$. Furthermore, the rela tive abundance of $P g$ was associated with the percentage of sites with active PDD in $\mathrm{CCP}+(\mathrm{p}=0.05)$ and $\mathrm{HC}(\mathrm{p}=0.04)$ but this was not seen for Aa (figure 1).

Conclusions: We report an increased prevalence of periodontal disease, $\mathrm{Pg}$ and $A a$ in anti-CCP positive at-risk individuals without synovitis. Interestingly, relative abundance of $\mathrm{Pg}$, but not $\mathrm{Aa}$, was associated with periodontitis, suggesting potential mechanistic differences that require further exploration. These data support the concept that periodontal inflammation and periodontopathic bacteria may both be important in the initiation of RA-related autoimmunity.

\section{REFERENCE:}

[1] Lundberg K. Nat Rev Rheumatol 2010

Disclosure of Interest: None declared

DOI: 10.1136/annrheumdis-2018-eular.5404

SATURDAY, 16 JUNE 2018

\section{Multi-disciplinary management of complex persistent pain}

\section{OP0353-HPR THE EFFECTS OF ARTHRITIS GLOVES ON HAND PAIN IN PEOPLE WITH RHEUMATOID OR INFLAMMATORY ARTHRITIS: A RANDOMISED CONTROLLED TRIAL (A- GLOVES TRIAL)}

A. Hammond ${ }^{1}$, Y. Prior ${ }^{1}$, C. Sutton ${ }^{2}$, S. Cotterill ${ }^{3}$, C. Heal ${ }^{3}$, E. Camacho $^{3}$, on behalf of The A-GLOVES research team. ${ }^{1}$ Centre for Health Sciences Research, University of Salford, Salford; ${ }^{2}$ Lancashire CTU, University of Central Lancashire, Preston; ${ }^{3}$ School of Health Sciences, Manchester University, Manchester, UK

Background: Arthritis (compression) gloves are commonly provided to people with rheumatoid arthritis (RA) and undifferentiated inflammatory arthritis (IA) in the UK health service. These apply pressure and warmth to relieve hand pain, stiffness and improve hand function. A systematic review identified little evidence to support their use.

Objectives: This randomised controlled trial tested effectiveness and cost-effec tiveness of mid-finger length compression (intervention) gloves (20\% Lycra: commonest glove model provided) with control gloves (i.e. oedema gloves: $11 \%$ Lycra: fitted at least one size too big) in people with RA and IA.

Methods: Both gloves, which had similar thermal qualities although the contro gloves did not provide compression, were provided by rheumatology occupational therapists, following training. ${ }^{2}$ Participants were also given brief advice on hand exercise and joint protection. Adults with RA/IA and persistent hand pain were randomised 1:1 to the two glove types, stratified by disease modifying anti-rheumatic drug (DMARD) change in previous 12 weeks. The primary outcome was dominant hand pain on activity Visual Analogue Scale (VAS:0-10); other outcomes included night hand pain, hand stiffness (both 0-10 VAS); Measure of Activity Performance Hand (MAP-HAND: 0-3). Multiple linear regression was undertaken to estimate the effect of group allocation on hand pain during activity, adjusting for the stratification variable and baseline values. Cost-effectiveness used individual patient level costs (intervention plus healthcare utilisation) and health benefit data (EQ-5D) to calculate costs and QALYs.

Results: 206 participants were randomised (103 to each glove type): median age 59 years [IQR 51,67]; women:166 (81\%); mean disease duration: 8.2 (SD 9.5) years; employed:76 (37\%); right hand dominant:185 (90\%). Of these, $163(79 \%)$ completed 12 week follow-up questionnaires. Both groups reported similar adherence to glove wear (mean 5.2 days/week). At $12 \mathrm{w}$, hand pain scores in both groups similarly improved: the between-groups mean difference of 0.1 was not statistically significant $(95 \% \mathrm{Cl}:-0.47$ to $0.67 ; \mathrm{p}=0.72)$. There were no significant differences between groups on any measures, with both groups improving similarly between baseline and 12 w. 73\% in both groups considered gloves beneficial. Intervention gloves had higher costs (£552 (SD £464); control £391 (SD $£ 543$ ) but comparable benefits to control gloves. Intervention gloves would cost $£ 83700$ to gain one QALY and were not likely to be cost-effective.

Conclusions: Compression (intervention) and loose-fitting arthritis (control) gloves had similar effects on hand pain, stiffness and function. Therefore, compression is not the 'active ingredient' in arthritis gloves. Loose fitting gloves providing warmth were perceived as equally effective by participants. We do not know if the therapist effect is important or whether ordinary gloves providing warmth would provide similar results.

\section{REFERENCES:}

[1] Hammond, et al. Clin Rehabil 2016 30:213-24

[2] Prior, et al. Rheumatology 2017. www.abstractsonline.com/pp8/\#!/4205 